\title{
Collision Avoidance in Restricted Visibility
}

\author{
Captain J. F. Wacher
}

In the January 1966 edition of this Journal Captain Wylie has given a lucid summary of the factors involved in the presentation on radar displays of true and relative motion. He also stated a conclusion on the combinations of display and ancillary equipment which he considers best satisfy the requirements for use when plotting to avoid collision at sea. Whilst acknowledging with respect all the work he has done to clarify the issues raised on this important but contentious subject over a number of years, a number of us has found it impossible to agree with his latest findings. As these may influence opinion in the shipping industry, the Board of Trade, the Courts of Law and the radar manufacturing industry, both at official and unofficial levels, they should not go unchallenged. Our disagreement concerns the priorities defined for display equipment and the method to be used for plotting. There can be no disagreement on academic principles.

Let us reconsider Captain Wylie's conclusions :

I (a) One display on relative motion with reflection plotter on which C.P.A. is obtained by direct plotting, whilst true courses and speeds are evaluated by the construction of velocity triangles also on the surface of the reflector.

This method is unsatisfactory for the following practical reasons:

(i) The limitations of the size of even the largest available display tubes and reflector plotters on the scale of a satisfactory plot.

(ii) Graphic errors inherent in the use of chinagraph pencils on concave reflector plotters superimposed over a radar display.

(iii) The difficulties at once involved in maintaining a multi-ship situation plot when 'rubbing out' relative to true-motion vectors becomes necessary, i.e. whenever any of the ships plotted alter course and a new set of velocity triangles have to be drawn.

(iv) The possibility of confusing the large number of constructed vectors inherent in this method-particularly in cases where several ships are approaching fine on each bow and are close together in bearing and/or range.

(v) The difficulties of overall situation assessment engendered by the interpretation of a number of vector triangles all close together on a reflector plotter-particularly for a fatigued observer.

2. Captain Wylie's (b) to (d).

(b) One display on true motion with transferred relative plot.

(c) Two displays one relative motion with reflector plotter, the other true motion.

(d) Two displays one Kelvin Hughes P.R.P. on relative motion, the other a true-motion display.

These arrangements are unsatisfactory for the following reasons:

(i) There is a greater likelihood of error where two sources rather than one have to be inspected before obtaining all the information needed to take a decision on collision avoidance. 
(ii) The likelihood of error is undoubtedly increased where the two sources to be inspected are shown respectively in true and relative motion.

3. Safe navigation and collision avoidance in restricted visibility requires the successful integration of a man-machine system incorporating radar displays and graphic plots. The effectiveness of such a system depends primarily on establishing proper blind navigation procedures for use on the bridge. These procedures must be carefully defined and need to be standardized. They necessarily involve efficient team work, formal observing, recording, plotting and reporting drills similar to those in daily use in airlines and in some merchant ships. Without the introduction of such exact systematic methods no proper control of the information obtained from radar displays and plots can be safely exercised. Their omission will result in further unnecessary collisions taking place. An attempt to define such procedures is to be found in this Journal. 1

4. When all aspects of radar usage, including safe navigation and collision avoidance, are considered in the context of the many different conditions encountered in the open sea, pilotage waters, rivers or harbour approaches, under varying traffic densities, then the total essential information:

(i) Own ship's true course and speed and future forecast position,

(ii) Other ships' risk of collision, i.e. C.P.A.,

(iii) Other ships' true course and speed and future estimated forecast position,

(iv) Effect on overall situation of own ship's alteration of course or speed,

(v) Own ship's navigational position.

is best shown on, and obtained from, the continuous use of a true plot.

5. This true plot must be constructed on a scale of not less than 1 in. $=1$ mile on a paper or perspex plotting board mounted adjacent to the radar display.

6. The use of a reflector plot superimposed on a radar display is seen to be primarily for other ship identification.

In conditions of very low traffic density, e.g. two or three ships presenting on the I 2-mile range scale, the reflector plotter may, however, be used for the construction of a true plot, whilst the main plot is being set up on the plotting board.

7. The practice of manual true plotting the maximum possible number of other ships, including non-immediate risks (which are readily identified by true plot), should always be followed: a corollary being that disregarding nonimmediate risks is a dangerous practice.

8. Of equipment at present available, the rapid photoplot true-motion radar display system alone enables a single operator to observe and plot the total navigational and collision avoidance situation in multi-ship situations as an integrated whole at a single display source of adequate scale.

9. Without the assistance of a semi-automatic plotting device, as above, safety in restricted visibility requires a team of two operators in addition to the captain.

10. The minimum essential equipment is :

\section{With one display}

A true, or relative motion, display stabilized north-up fitted with a reflector plotter, with a manual plotting board mounted on a table alongside the radar. The method to be used is manual with two operators, a scanner and a plotter. Continual surveillance of the radar display must be maintained by the scanner, 
who identifies other ships with designation letters on the reflector plot, and passes true bearing and range information to the plotter. The plotter constructs a true plot on the plotting board, from which source the captain obtains all the information necessary to take a decision on the total situation for collision avoidance, i.e. items (i) to (iv) listed in (4) above. Navigational information in this system has to be obtained from the display scanner for transfer to the navigational chart. There is no reason why the captain should not take the duty of scanner. Ideally all officers should be trained in this most exacting task.

The answer to the question of the availability of manpower is axiomatic. Team work is essential to safe radar blind navigation, and if the safety of the ship requires double manning for short periods then this must be carried out.

I . With two displays

(a) A true- or relative-motion display stabilized north-up with reflector plotter superimposed on the display. (This display to be used in a similar manner to the single display system described above in the event of any failure of the second display.)

(b) Photographic rapid plot. This semi-automatic device enables one operator to construct a true plot of own and all other ships observed swiftly, and to coordinate the navigational situation, which is also presented, so that navigation and collision avoidance decisions can be taken by inspection of one source, i.e. items (i) to (v) listed in (4) above. In addition, this system keeps a permanent, continuous record on film of all navigation and collision avoidance situations encountered accurately related to time.

The number of radar simulator course certificates issued by Sir John Cass College is now 1320 , and Captain M. W. M. Weekes, who is in charge of the course, confirms that $9^{8}$ per cent of practising seafarers, after being taught both relative and true plotting, continue to conclude that true plotting is the method which will best give them collision avoidance information. In addition, practical experience at sea since 1955- during which time both true and relative type plots have been evaluated in vessels ranging from 8000 to 29,000 tons operating on world-wide routes-has confirmed our view that the best method of plotting for general use in all situations is as described above.

The evidence, from those in charge of and attending simulator courses, and from a deal of practical experience at sea, shows that the implementation of standard and simplified bridge radar blind navigation procedures, using a true plot, would greatly assist in reducing the likelihood of collision in restricted visibility.

It is time for the Board of Trade to instigate a professional study in depth-by practising seamen as well as by administrators- - to simplify and standardize such procedures which will result in definitive rather than advisory notices. These are at present clearly unsatisfactory in so far as collisions in restricted visibility continue with unhappy frequency.

\section{REFER E N CE}

1 Why ships collide (a discussion). This Journal, 16, 343 . 